\title{
ANFIS based Information Extraction using K-means Clustering for Application in Satellite Images
}

\author{
Ricky Gogoi \\ Department of Electronics and Communication \\ Technology \\ Gauhati University
}

\author{
Kandarpa Kumar Sarma \\ Department of Electronics and Communication \\ Technology \\ Gauhati University
}

\begin{abstract}
Information extraction from satellite images is a challenging task. This is because of the associated uncertainty arising out of improper capture and subsequent transfer. Fuzzy systems are suitable for such applications because of the fact that these have the ability to capture minute variations in the patterns presented. Fuzzy systems are expert decision making tools that require support from Artificial Neural Network (ANN) for inference generation. This leads to the formation of NeuroFuzzy system (NFS). A NFS requires certain apriori information for making appropriate decision. Apriori knowledge can be provided manually but it becomes tedious, hence certain computational approaches are required. In this paper, the focus is given on the development of an information extraction system based on K-means clustering (KMC) and ANN and an adaptive neuro- fuzzy inference system (ANFIS) based system with the same purpose to achieve enhanced performance as compared to each other. We specially deal with an ANFIS aided by KMC for use with information extraction from satellite images. Experimental results show that such system is fully automatic and effective in dealing with information extraction from river images with forest and sand distribution along its banks.
\end{abstract}

\section{General Terms}

Adaptive Neuro Fuzzy System, Neuro Fuzzy System, Artificial Neural Network, K-means clustering.

\section{Keywords}

ANFIS, NFS, Fuzzy system.

\section{INTRODUCTION}

Information extraction from satellite images is a tedious task because variation of certain patterns gives rise to uncertainty in decision making. Due to microscopic changes which are as common during the capturing phase of satellite images, information extraction provides a lot of tricky situations and requires delicate system design for effective performance. To study a particular topology, it is important to separate out specific patterns from the other part of the image. Such segmentation of the regions of interest (ROI) can be performed by using $\mathrm{K}$-means clustering (KMC) technique where cluster portions are required to be identified [1] [2]. The Artificial Neural Networks (ANN) based approaches are capable of learning from model free environment and can be used for capturing information from data segments generated by KMC algorithm to define the required pattern in an image. Since the image may consist of various patterns of a particular object, it becomes difficult to extract a particular pattern from the whole image. In this type of case use of fuzzy logic helps to get various patterns of a particular object as a whole in a single cluster. The ANN based approach along with the fuzzy logic can predict, from an applied pattern, the required portion and provide near approximate ROI segments from satellite images. The hybrid system formed by KMC and ANN is able to predict portions from a model free environment. On the other hand a hybrid system formed by fuzzy logic and ANN can be applied to capture patterns with finite changes in an environment full of uncertainty from a satellite image [3]. In this paper, the focus is given on the development of an information extraction system based on KMC and ANN and an Adaptive Neuro Fuzzy System (ANFIS) based system with the same purpose to achieve enhanced performance as compared to each other. We specially deal with an ANFIS aided by KMC for use with information extraction from satellite images. Experimental results show that such system is effective in dealing with information extraction from river images with forest and sand distribution along its bank.

\section{THEORITICAL BACKGROUND}

The KMC is a technique mostly used often to group samples with certain similarity. Initially, random selection of points those are likely to be in different clusters gives the centroids for separate clusters. With these centroids the remaining points of that particular image forms the cluster as given by the user. The use of ANN enables the system to examine the cluster in a particular image and finally train and provides the required form with a number (defined by the user) of sections in an image.

The ANFIS system is formed by a neuro- fuzzy system (NFS) which is a combination of ANN and Fuzzy systems. Also, with the NFS, is related an inference engine which helps in making appropriate discrimination between applied patterns. The entire mechanism is adaptive for which it can track subtle variations in the input patterns. We here briefly cover the basic theoretical notions related to the system which is appropriately modified for information extraction from satellite images.

\subsection{K- means Clustering}

Clustering is an unsupervised learning technique and is the collection of similar type of objects into a single group as shown in Figure 1. There are various types of clustering techniques among which $\mathrm{KMC}$ is the most commonly and popularly used where $\mathrm{K}$ is the number of cluster centers defined by the user. Different steps for the algorithm for the $\mathrm{KMC}$ are as follows: 
- Initialization of the number of clusters $\mathrm{K}$, cluster centres for $\mathrm{K}$ clusters $\mu_{1} \mu_{2}, \ldots \ldots \ldots \ldots . . \mu_{K}$, and among the points $\mathrm{n}$;

- Classification of $\mathrm{n}$ samples according to the nearest $\mu_{i}$, where $\mathrm{i}$ is the number of clusters;

- $\quad$ Recomputation of $\mu_{i}$ till it stops further calculation;

- $\quad$ Return the clusters $\mu_{1,} \mu_{2}, \ldots \ldots \ldots \ldots . . . \mu_{K}$,

The KMC is simple technique and works in almost all cases of classification $[4,5]$.

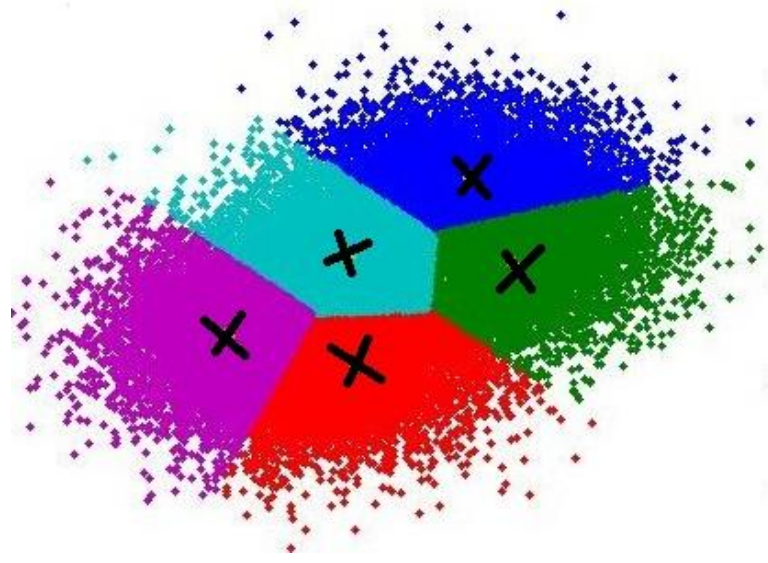

Fig 1: Depiction of KMC

\subsection{Artificial Neural Network}

The human brain is an intelligent system. Artificial intelligence is developed based on the logical and structured characteristics of the human brain. Researchers developed a novel system that works like biological nervous system called Artificial Neural Network (ANN) [6].

Generally, all ANNs consist of minimum three layers as shown in the Figure 2, connected to each other in which the first, is the input layer and receives the information from external sensors and passes to the hidden layers which may contain multiple blocks interconnected to each other in the form of neurons. The resultant output is passed to the real world through the output layer.

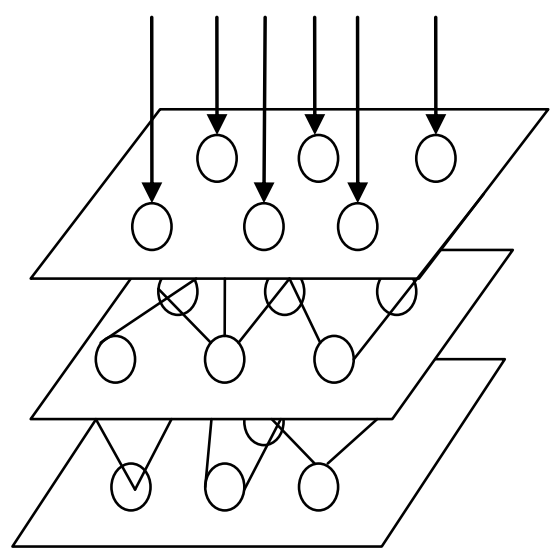

Fig 2: Artificial Neural Network

\subsection{Fuzzy Systems}

In 1965, L. A. Zadeh introduced the concept of Fuzzy Logic based on which subsequent ideas as well as systems have been developed. Complexity, credibility and uncertainty are the three major characteristics based on which a fuzzy system are deployed. Membership grades define the degree of fuzzyness in fuzzy sets. Fuzzy system applies the logic related to fuzzy sets [7].

\subsection{Neuro -Fuzzy System}

It is a system in which the parameters in fuzzy form based on are applied to a learning algorithm based on ANN concepts. This type of system is always based on fuzzy rules and was an inference mechanism [8].
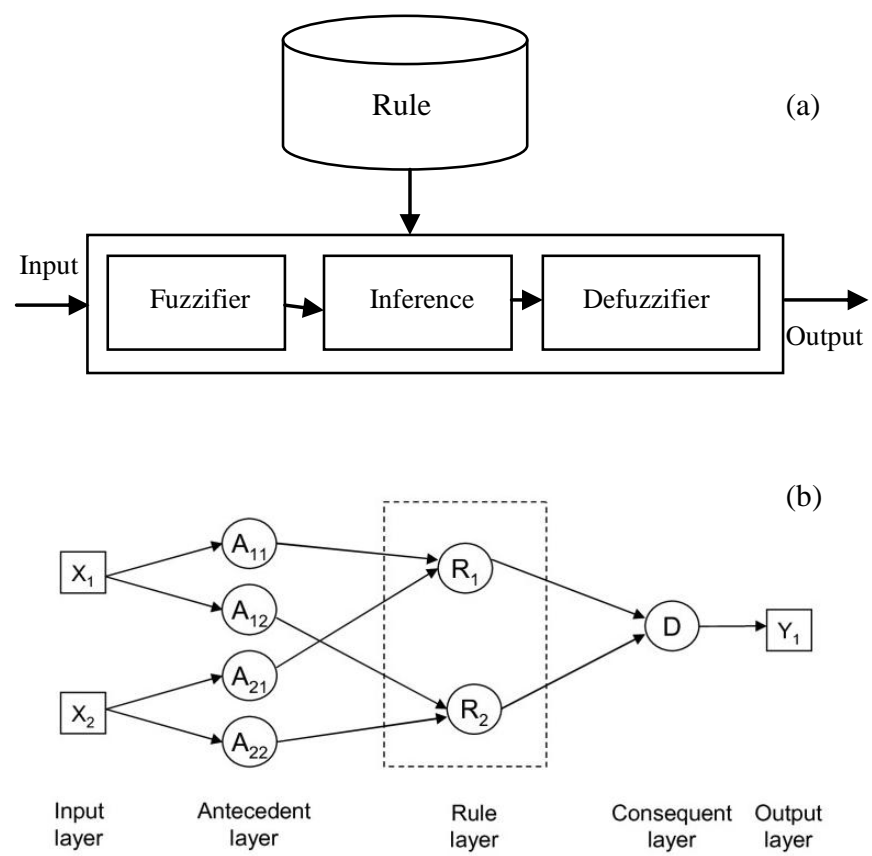

Fig 3: Mamdani type FIS

\subsection{Inference Engine}

The inference system is specific program of an expert system which runs from the rules already contained in the knowledge base using artificial intelligence techniques such as ANN [9].

\subsection{ANFIS}

These are a collection of adaptive networks which function equivalently as fuzzy inference systems. The process of fuzzy inference system (FIS) formulates the mapping of a given input to an output by using fuzzy logic. The FIS involves three main processes as membership functions, logical operations, and If-Then Rules. FIS can be successfully implemented in many systems [10, 11, 12]. It has a multidisciplinary nature by which it can be implemented anywhere to control, to predict, to make an expert system etc. In FIS, inputs are either in fuzzy or crisp forms for which it results an outputs which are in fuzzified form. It sometimes requires crisp results. A defuzzification process gives the required result in crisp form. The fuzzy if- then rules describes the behavior of the FIS. A complete FIS is shown in Figure. 3 . 
There are two types of FIS: Mamdani-type and Sugeno-type. Depending upon the way outputs are determined, these two types of inference systems vary in their nature.

The Mamdani FIS was first proposed by E.H. Mamdani at the University of London in 1974. It applies the system logic in a system controlled by fuzzy logic. This type of FIS results the output membership functions as fuzzy sets which needs defuzzification process to get the result in crisp form. Depending upon the number of inputs and number of outputs along with the maximum number of rules a Mamdani type FIS model can be constructed with definite grades of membership functions. A Mamdani NFS (Figure 3) uses a supervised learning technique which is a back propagation learning to retain the variations in the parameters of the membership functions. There are different layers of this type of system as:

- Input Layer: Each node represents one input variable which only transmits input to the next layer.

- Fuzzification Layer: Each node represents one label to one of the input variable of first layer. The output link of this layer represents the membership value. This layer specifies the degree to which an input value belongs to a fuzzy set. A clustering algorithm determines the number and type of membership functions.

- Rule Antecedent Layer: The nodes in this layer represents the type of operator (T-norm operator) used in this node. The output of this layer results the strength of the corresponding fuzzy rule.

- Rule Consequent Layer: This node combines the incoming rule antecedents and determines the degree to which they belong to the output label. The number nodes here are equal to the number of rules.

- Combination and Defuzzification Layer: The combination of all rules consequents is done in this node and finally after defuzzification it computes the crisp output.

Takagi, Sugeno and Kang proposed the Sugeno Fuzzy model when they were supposed to develop a systematic approach for generating fuzzy rules from a given input- output data set $[13,14]$. In general, Sugeno-type systems can be used to model any inference system in which the output membership functions are either linear or constant $[15,16]$.
This model uses a mixture of back propagation and least mean square estimation. Different layers of this system model are as follows:

- Input Layer, Fuzzification Layer and Rule Antecedent Layer: These three layers function as the Mamdani type NFS model.

- Rule Strength Normalization: Every node in this layer calculates the ratio of the $i^{-{ }^{\text {th }}}$ rule's strength to the sum of all rules strength,

$$
\boldsymbol{W}=\frac{\mathbf{W}}{\boldsymbol{M}_{\mathbf{1}}+\boldsymbol{w}_{2}} \quad \mathrm{i}=1,2,3 \ldots
$$

- Rule Consequent Layer: Every node i in this layer is with a node function,

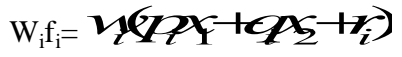

- Rule Interference Layer: This layer has a single node. It computes the overall output as the summation of all incoming signals and is expressed as,

$$
\text { Overall output }=\sum_{i} v f_{i}=\frac{\sum_{i} v y_{i}}{\sum_{i} v_{i}}
$$

\section{PROPOSED SYSTEM MODEL}

The system model of the proposed ANFIS-based system is shown in Figure 4. The system model for the proposed KMC based system is shown in Figure 5.

The first part of both the works is pre-processing. A sequence of steps like noise removal or addition (in case of noisy image processing), enhancement, reshaping and sharpening is performed to make the considered image for subsequent processing $[17,18,19]$.

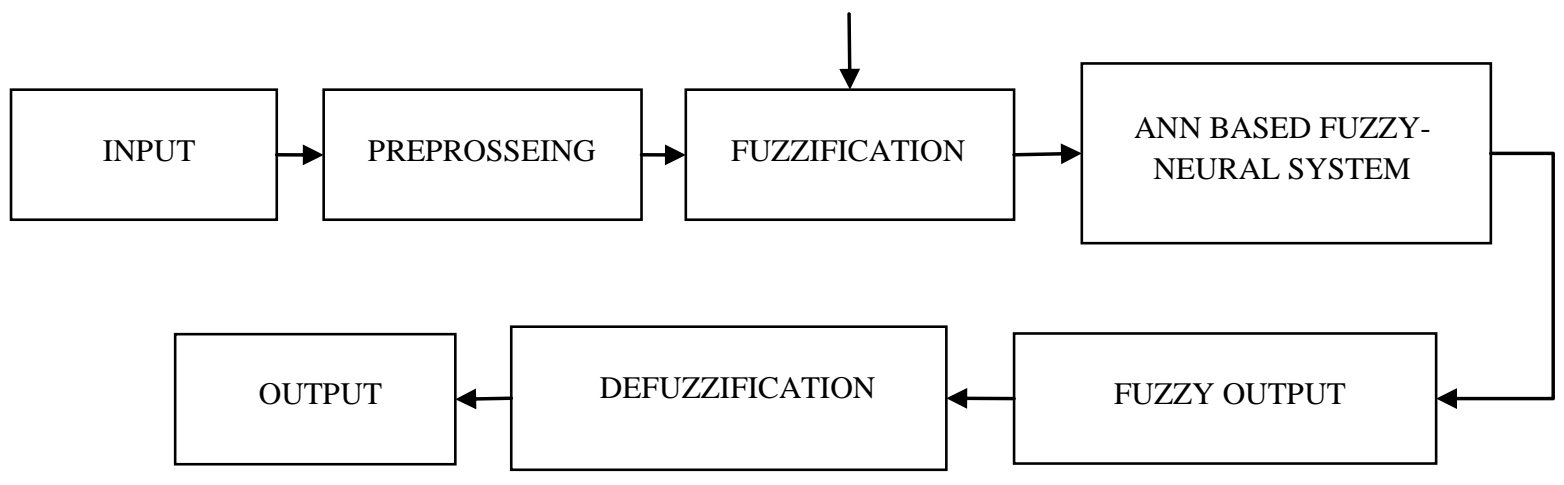

Fig 4: System model using ANFIS 


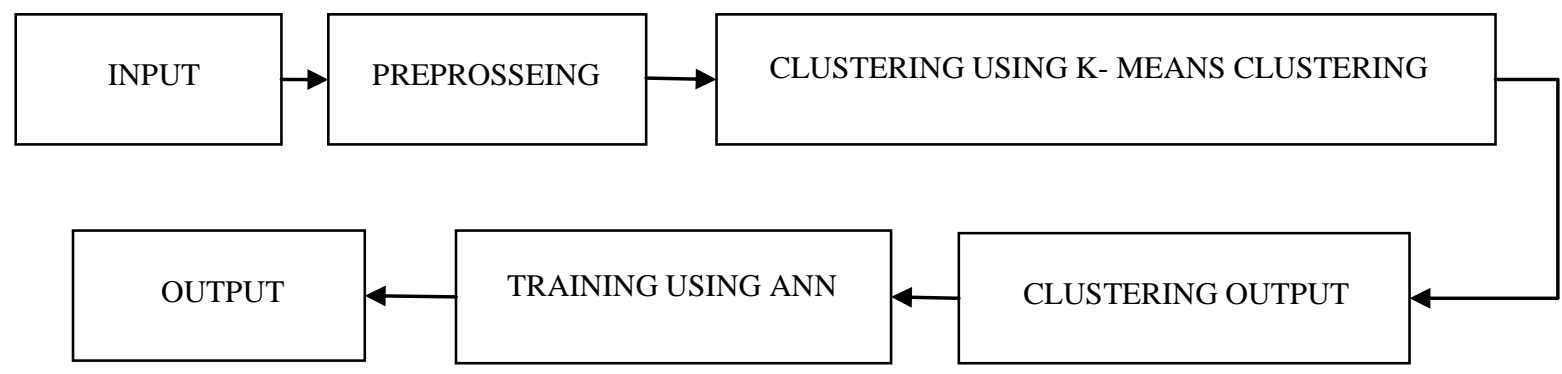

Fig 5: System model using KMC

There are various types of clustering techniques based on which a number of given objects or data can be classified. It is an unsupervised learning process. The KMC can be done based on the similarity between the elements of the same cluster and dissimilarity between the other clusters. The Euclidian distance vector is used to find the distance between the neighbourhood points to form the clusters. The first step is user defined where user will specify the number of clusters along with the cluster centre (centroid) values and the collection of object. In the second step, the distance measures are found using the value of the centroid. It will work until the last point which here is the last pixel value of the image. The next step is the result, where the clusters formed by the system will be generated. Here only gray scale images are used to do the clustering.

As already mentioned there are two types of FIS: Mamdanitype and Sugeno type. Depending upon the way outputs are determined these two types of inference systems vary in their nature $[20,21]$.

Table 1. CONFIGURATIONS OF ANFIS AND KMEANS CLUSTERING

\begin{tabular}{|c|c|}
\hline Item & Descriptions \\
\hline \multicolumn{2}{|c|}{ ANFIS } \\
\hline $\begin{array}{c}\text { Data structure of the input } \\
\text { image }\end{array}$ & $\begin{array}{c}\text { Double precision grayscale } \\
\text { image }\end{array}$ \\
\hline Input data size & $250 \times 375$ \\
\hline Membership type & $\begin{array}{l}\text { Bell- shaped membership } \\
\text { function }\end{array}$ \\
\hline $\begin{array}{l}\text { Number of membership } \\
\text { function }\end{array}$ & Six \\
\hline Type of ANFIS & Sugeno type ANFIS \\
\hline \multicolumn{2}{|c|}{ KMC } \\
\hline $\begin{array}{c}\text { Data structure of the input } \\
\text { image }\end{array}$ & $\begin{array}{c}\text { Double precision greyscale } \\
\text { image }\end{array}$ \\
\hline Input data size & $250 \times 375$ \\
\hline Number of clusters & 2 \\
\hline
\end{tabular}

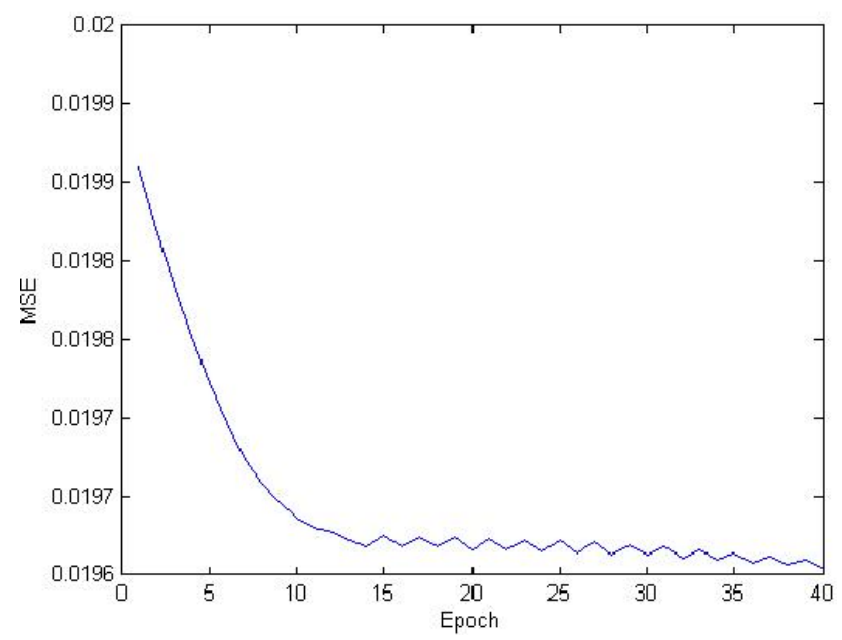

Fig 6: MSE convergence with epoch of the ANFIS

\section{EXPERIMENTAL RESULTS}

Here we have used the Sugeno-type FIS. This type of system uses a combination of the least-squares method and the back propagation gradient descent (BPGD) method for training. In the training phase, the input matrix contains the input as well as the output vectors. The last column contains the output data and the other column contains input. The FIS provides the number and type of membership function according to which the training process starts. The steps used in the system are briefly discussed below:

The first step compares the input variables with the membership functions to obtain the membership values. Next, the membership values on the targeted part are combined to get the weight of each rule. Finally, the qualified consequent for each rule is generated depending upon the weight. Experiments are carried out with an ANFIS network and a KMC network with configuration parameters shown in Table 1. Training convergence of the ANFIS is shown in Figure 6.

The ANFIS system is fast but distinguishes only the few numbers of clusters specified manually. But the KMC network doesn't require manual intervention and performs the clustering task automatically provided the cluster centers are clearly identified. But computationally, the second approach is more stringent. It takes more time to respond. 
Table 1 shows certain training time performance of the ANFIS system. The mean square error (MSE) for different epochs and time taken are shown in Table 2.

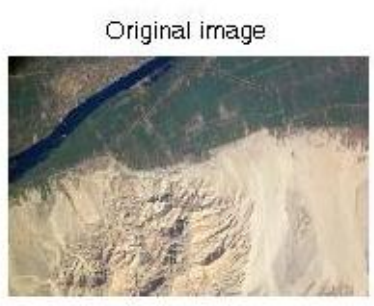

Target image

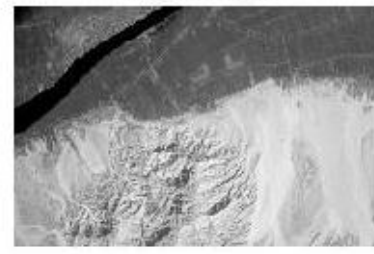

Fig 7: Images used to test and train ANFIS

Table 2. MSE AND TIME TAKEN FOR DIFFERENT EPOCHS

\begin{tabular}{|c|c|c|c|}
\hline SI No. & Epochs & MSE X 10 & Time in seconds \\
\hline 1 & 10 & 4.32 & 48.11 \\
\hline 2 & 50 & 4.55 & 103.17 \\
\hline 3 & 100 & 4.61 & 171.28 \\
\hline 4 & 200 & 4.62 & 311.68 \\
\hline
\end{tabular}

Further, the clustering technique makes clear demarcation between the important and insignificant regions. The demarcation also handles multiple clusters automatically which in case of the ANFIS system requires manual intervention. Speed wise, the ANFIS system is faster but has limited demarcation capability. Moreover, the KMC algorithm has better discrimination capability which helps in extracting the required portion of the information. Figures 7 and 8 are the results of the systems using ANFIS and KMC. As seen in Figure 7 , while extracting river or water body information from the satellite image, the ANFIS system provided both significant and non-significant data. The useless portion of the data cannot be removed from the ANFIS output. But the significant portion has high precision which is useful. This is seen from the ANFIS generated image where the river is extracted along with certain portions of the river bank as well. But the KMC technique extracts only the river and related water body section of the image. It removes the rest of the portion which is an advantage. The strength of the proposed system is thus obvious.

\section{CONCLUSION}

Here, we have described the design of an ANFIS model and a system using KMC configured for information extraction from satellite images. An image with special focus on river side content captured by satellite is used. The framework formulated is, however, in the present form limited only to gray-scale images. It can be extended to include multidimensional colour images as well which shall make it an effective tool for the study of a range of river and topography related phenomena based on satellite photography. The experiments performed show that both the system works for satellite images. The time taken by KMC is more as compared to the ANFIS based system. Since the KMC takes more time, it is not feasible to use it in real time process and requires modification including the use of specialized hardware. As fuzzy systems give more precision in microscopic variations, the ANFIS based system shall be more effective if inference making is combined with the apriori knowledge generated by KMC.

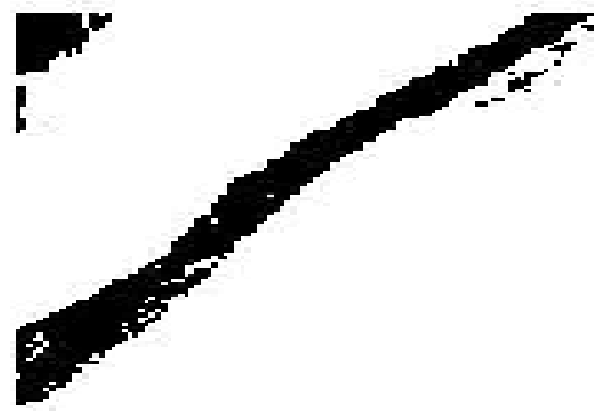

Fig 8: KMC resulted image

\section{REFERENCES}

[1] Joaquin Perez Ortega, Ma. Del Rocio Boone Rojas, Maria J. Somodevilla Garcia, "Research issues on Kmeans Algorithm: An Experimental Trial Using Matlab".

[2] Ng H. P., Ong S. H., Fung K. W. C., Goh P. S., Nowinski W. L., 'Medical Image Segmentation Using KMeans Clustering and Improved Watershed Algorithm", IEEE, 2006, pp 61- 65 .

[3] Jang J S R, Sun C T and Mizutani E, Neuro-fuzzy and soft computing a computational approach to learning and machine intelligence, PHI Learning Private Limited, 2011.

[4] Vijaya R. Saravanan AM, Jothi Venkateswaran C, Clustering Technique Using K-means Dempster- Shafer Theory of Evidence, Indian J. Edu. Inf. Manage., Vol. 1, No.5 (May, 2012), pp. 223-227

[5] Pattern Classification: Richard O. Duda, Peter E. Hart, David G. Stork, $2^{\text {nd }}$ Edition, Wiley India, 2007

[6] Haykin. S, Neural Networks A Comprehensive Foundation, $2^{\text {nd }}$ edition, Pearson Education, New Delhi, 2003.

[7] L A Zadeh: Fuzzy sets: Information and Control: pp $8: 338-353,1965$

[8] Fuller, Robert: Introduction to Neuro-Fuzzy Systems,Physica-Verlag, A Springer Verlag Company, 2000

[9] Victor Boskovitz, Hugo Guterman: An Adaptive NeuroFuzzy System for Automatic Image Segmentation and Edge Detection: IEEE Transactions on Fuzzy Systems: V01. 10, NO. 2, April 2002, pp. 247- 262

[10] Michael Makridis, Nikolaos E. Mitrakis, Nikolaos Nikolaou and Nikolaos Papamarkos: Text Extraction Using Component Analysis and Neuro-fuzzy Classification on Complex Backgrounds:A. Heyden and 
F. Kahl (Eds.): SCIA 2011, LNCS 6688, pp. 742751, 2011: Springer- Verlag Berlin Heidelberg, 2011

[11] Andrea Baraldi, Elisabetta Binaghi, Palma Blonda, Pietro A Brivio, and Anna Rampini: Comparison of the Multilayer Perceptron with Neuro- Fuzzy Techniques in the Estimation of Cover Class Mixture in Remotely Sensed Data: IEEE Transactions on Geoscience and Remote Sensing, Vol. 39, No. 5, May, 2001

[12] Massimo Panella, Antonio Stanislao Gallo: An Input Output Clustering Approach to the Synthesis of ANFIS Networks: IEEE Transactions on Fuzzy Systems: Vol. 13, NO. 1, pp. 69-81,February, 2005

[13] H J Zimmermann: Fuzzy set theory and its applications, Fourth Edition: Springer, 2011

[14] E H Mamdani: Application of fuzzy algorithms for control of simple dynamic plant: Proc. Inst. Elec. Eng.: pp 121 1585-1588: 1974
[15] George J Klir and Bo Yuan: Fuzzy Sets and Fuzzy Logic Theory and Applications: Prentice Hall of India, New Delhi, 2008

[16] M Sugeno, G T Kang: Structure identification of Fuzzy model: Fuzzy sets and Systems: 28:15-33, 1988

[17] T Takagi, M Sugeno: Fuzzy identification of systems and its applications to modeling and control: IEEE Transactions on Systems, Man, and Cybernetics: 15:116132,1985

[18] K Guney, "Comparison of Mamdani and Sugeno Fuzzy Inference System models for resonant frequency calculation of rectangular microstrip antennas" Progress In Electromagnetics Research B: Vol. 12, 81104,

[19] Lamba V K, Neuro Fuzzy Systems, University Science Press, New Delhi

[20] Timothy J Ross, Fuzzy Logic with Engineering Applications, Second Edition: Wiley India, 2008

[21] H.J. Zimmermann, Fuzzy Set Theory and its applications, Fourth Edition, Springer 